\title{
Diversity, Virulence, and 2,4-Diacetylphloroglucinol Sensitivity of Gaeumannomyces graminis var. tritici Isolates from Washington State
}

\author{
Youn-Sig Kwak, Peter A. H. M. Bakker, Debora C. M. Glandorf, \\ Jennifer T. Rice, Timothy C. Paulitz, and David M. Weller
}

First and fourth authors: Department of Plant Pathology, Washington State University, Pullman, WA 99164-6430; second author: PlantMicrobe Interactions, Department of Biology, Utrecht University, Padualaan 8, $3584 \mathrm{CH}$ Utrecht, The Netherlands; third author: GMO office, Expert Centre of Substances, National Institute of Public Health and the Environment, P.O. Box 1, 3720 BA Bilthoven, The Netherlands; and fifth and sixth authors: USDA-ARS, Root Disease and Biological Control Research Unit, Pullman, WA 99164-6430. First, second, third, and sixth authors contributed equally to this work. Accepted for publication 25 January 2009.

\begin{abstract}
Kwak, Y.-S., Bakker, P. A. H. M., Glandorf, D. C. M., Rice, J. T., Paulitz, T. C., and Weller, D. M. 2009. Diversity, virulence, and 2,4-diacetylphloroglucinol sensitivity of Gaeumannomyces graminis var. tritici isolates from Washington State. Phytopathology 99:472-479.

We determined whether isolates of the take-all pathogen Gaeumannomyces graminis var. tritici become less sensitive to 2,4-diacetylphloroglucinol $(2,4-\mathrm{DAPG})$ during wheat monoculture as a result of exposure to the antibiotic over multiple growing seasons. Isolates of $G$. graminis var. tritici were baited from roots of native grasses collected from noncropped fields and from roots of wheat from fields with different cropping histories near Lind, Ritzville, Pullman, and Almota, WA. Isolates were characterized by using morphological traits, G. graminis variety-specific
\end{abstract}

ABSTRACT polymerase chain reaction and pathogenicity tests. The sensitivity of $G$. graminis var. tritici isolates to 2,4-DAPG was determined by measuring radial growth of each isolate. The $90 \%$ effective dose value was 3.1 to $4.4 \mu \mathrm{g} \mathrm{ml}-1$ for 2,4-DAPG-sensitive isolates, 4.5 to $6.1 \mu \mathrm{g} \mathrm{m} l^{-1}$ for moderately sensitive isolates, and 6.2 to $11.1 \mu \mathrm{g} \mathrm{ml}{ }^{-1}$ for less sensitive isolates. Sensitivity of G. graminis var. tritici isolates to 2,4-DAPG was normally distributed in all fields and was not correlated with geographic origin or cropping history of the field. There was no correlation between virulence on wheat and geographical origin, or virulence and sensitivity to 2,4-DAPG. These results indicate that G. graminis var. tritici does not become less sensitive to 2,4-DAPG during extended wheat monoculture.

Additional keywords: biological control, Pseudomonas fluorescens, take-all.
Take-all, caused by Gaeumannomyces graminis (Sacc.) von Arx \& Olivier var. tritici Walker (G. graminis var. tritici), generally develops at a soil $\mathrm{pH}$ of 5.5 to 8.5 and where wheat is grown under moist conditions $(7,9,19,22,29)$. As a result, take-all is common throughout the world and is considered the most important root disease of wheat $(7,10,22)$. G. graminis var. tritici survives saprophytically as mycelium in dead roots, crowns, and tiller bases, the inoculum source for the next crop $(9,19,29)$. Primary infection of roots of seedlings occurs with the growth of dark runner hyphae on the root surface. Hyaline hyphae penetrate into the cortex and colonize the vascular tissue, causing characteristic black lesions. Runner hyphae continue to grow over the root surface to other roots and upward to the crown and stem bases. Early infection of the plant ultimately causes yellowing of lower leaves, stunting, and premature death of plants in patches. Wheat is highly susceptible to take-all, but other members of the family Poaceae (i.e., barley, rye, and triticale) are also infected $(9,19)$.

Crop rotation and tillage are effective controls of take-all; however, the trends are toward less tillage and growing two or three wheat crops before a break, and these practices exacerbate the disease. Take-all is also controlled by take-all decline (TAD), the spontaneous decrease in take-all incidence and severity induced by continuous wheat or barley monoculture after a severe out-

Corresponding author: D. M. Weller; E-mail address:wellerd@wsu.edu

doi:10.1094/PHYTO-99-5-0472

This article is in the public domain and not copyrightable. It may be freely reprinted with customary crediting of the source. The American Phytopathological Society, 2009. break of the disease $(12,22,39)$. In the Pacific Northwest of the United States and in The Netherlands, TAD develops because of the build-up of populations of 2,4-diacetylphloroglucinol $(2,4$ DAPG)-producing Pseudomonas fluorescens ( $p h l D^{+}$) and production of the antibiotic in the rhizosphere during wheat and barley monoculture $(32,33,38,39)$. The 2,4-DAPG biosynthetic locus includes the operons phlACBDE and phlF that function in synthesis, export, and regulation (2). 2,4-DAPG accumulation in the wheat rhizosphere is proportional to the population density of phlD ${ }^{+}$P. fluorescens at densities above $10^{5}$ colony forming units (CFU) $\mathrm{g}^{-1}$ of root, and 2,4-DAPG concentration per $p h l D^{+}$population unit is constant $\left(0.62 \mathrm{ng} / 10^{5} \mathrm{CFU}\right)$ (31). Roots of wheat grown in a Quincy TAD soil supported $4.3 \times 10^{6} \mathrm{CFU}$ of phlD isolates and $19.1 \mathrm{ng}$ of 2,4-DAPG g ${ }^{-1}$ of root (31).

Among all soilborne pathogens common in Pacific Northwest wheat fields, G. graminis var. tritici is the most sensitive to 2,4DAPG $(1,24)$. Mazzola et al. (24) screened G. graminis var. tritici isolates from seven countries and seven states and showed differential sensitivity to 2,4-DAPG. Isolates that were not inhibited by 2,4-DAPG at $3 \mu \mathrm{g} / \mathrm{ml}$ were not suppressed by $p h l D^{+} P$. fluorescens strain Q2-87 on wheat roots, whereas isolates sensitive to this concentration were controlled. The genetic variation and diversity known to exist within the take-all pathogen, even though sexual recombination is not a common part of its ecology, apparently are reflected in the differences among $G$. graminis var. tritici isolates in sensitivity to 2,4-DAPG.

TAD occurs worldwide $(22,35,36)$ and follows a similar pattern everywhere, yet the speed of its development and the robustness and longevity of the suppressiveness vary among fields (22). The long-term goal of our research is to identify biotic and abiotic factors that impact the robustness of take-all suppressiveness in 
order to maximize the effectiveness of TAD for growers who crop wheat continuously or in rotation with barley. The specific objectives of this study were to determine the range of sensitivities to 2,4-DAPG among isolates of $G$. graminis var. tritici from TAD and non-TAD soils and to determine whether G. graminis var. tritici isolates with reduced sensitivity to 2,4-DAPG become enriched in wheat monoculture soil as a result of exposure to the antibiotic over multiple growing seasons.

\section{MATERIALS AND METHODS}

Isolates, media, and storage. Six strains of G. graminis var. tritici were used as controls throughout this study: R3-111a-1, ARS-A1, MV-116, MV-119, L-109, and L-116 (14). R3-111a-1 originally was isolated in 1980 from wheat grown in a TAD field near Moses Lake, WA. ARS-A1 was isolated in 1990 from wheat grown on the USDA-ARS Palouse Conservation Field Station near Pullman. MV-116 and MV-119 were isolated in 1986 from wheat grown on the Washington State University (WSU) Mount Vernon Northwestern Washington Research \& Extension Center, Mt. Vernon, WA. L-109 and L-116 were isolated in 1986 from the TAD field at the WSU Lind Dryland Research Station, Lind, WA.

G. graminis var. tritici isolates were routinely cultured on onefifth strength potato dextrose agar (1/5× PDA): potato dextrose broth (PDB, 4.5 g) (BD, Sparks, MD), agar (20 g) (Sigma Chemical Co., St. Louis, MO), and water (1 liter). The G. graminis var. tritici semiselective medium (R-PDA) (14) was used to isolate $G$. graminis var. tritici from roots. For R-PDA, peeled potato slices $(40 \mathrm{~g})$ were boiled for $10 \mathrm{~min}$ and the filtered extract was increased to a volume of 1 liter with water. Dextrose $\left(4 \mathrm{~g} \mathrm{liter}^{-1}\right)$ and agar (18 $\mathrm{g} \mathrm{liter}^{-1}$ ) were added and the $\mathrm{pH}$ of the medium was adjusted to 6.5 before autoclaving. Rifampicin $\left(100 \mu \mathrm{g} \mathrm{ml} \mathrm{m}^{-1}\right)$ (Sigma Chemical Co.) and Rizolex (Tolclofos-Methyl; purity 98\%) $\left(1 \mu \mathrm{g} \mathrm{ml}^{-1}\right)($ Chem Service, West Chester, PA) were added after autoclaving when the medium cooled to below $60^{\circ} \mathrm{C}$.

Isolates were stored by a variety of methods. R3-111a-1 and ARS-A1 were stored on 1/5x PDA amended with rifampicin (100 $\mu \mathrm{g} \mathrm{ml}^{-1}$ ) at $4^{\circ} \mathrm{C}$ and once a year isolates were taken out of storage, cultured on 1/5x PDA, and tested for virulence on wheat as described below. Each isolate was reisolated from diseased roots, grown on $1 / 5 \times$ PDA with rifampicin, and again placed at $4^{\circ} \mathrm{C}$. Isolates MV-116, MV-119, L-109, and L-116 were stored in glycerol as hyphal fragments at $-80^{\circ} \mathrm{C}$. G. graminis var. tritici isolates collected during this study were stored at room tempera- ture on slants of $1 / 5 \times$ PDA, with and without mineral oil, and as colonized $1 / 5 \times$ PDA agar plugs in autoclaved distilled water (15).

Isolation and culture conditions of $G$. graminis var. tritici. $G$. graminis var. tritici was isolated from wheat grown in fields in eastern and central Washington State that had undergone at least 3 years of wheat or barley monoculture or crop rotation. $G$. graminis var. tritici was also isolated from native grasses growing in noncropped (virgin) fields. Table 1 shows a list of the fields from which $G$. graminis var. tritici isolates were collected. The Lind TAD (LD) field is located on the WSU Lind Dryland Research Station (33). LD isolates were from healthy looking wheat randomly selected throughout the field. LDP isolates were also from the Lind TAD field but were isolated from wheat with symptoms of take-all within distinct take-all patches. The Lind virgin (LV) site has never been cropped and is located about $100 \mathrm{~m}$ from the Lind TAD field (33). The Pullman conducive field (PC) (32) is located on the USDA-ARS Palouse Conservation Field Station near Pullman. The Almota TAD (ADB) field is located on a commercial farm near the Port of Almota and is direct seeded. The Ritzville TAD (RD) field is located on a commercial field near Ritzville, WA, and is irrigated.

Most isolates were collected by using a baiting method and a tube assay essentially as described by Cook and Naiki (11). Roots were washed under running tap water to remove soil and then blotted dry with a paper towel. Roots from a single plant were excised, rolled into a ball, and placed on the top of a 6-cm column of autoclaved vermiculite inside a plastic tube $(15 \mathrm{~cm}$ long, $2.5 \mathrm{~cm}$ diameter, Stuewe and Sons Inc., Corvallis, OR); 100 tubes were hung in a plastic rack. The roots were covered with a $1-\mathrm{cm}$ layer of autoclaved vermiculite; three wheat seeds (spring wheat cv. Penawawa) were sown and then covered with a 1-cm layer of vermiculite. Each tube received $12 \mathrm{ml}$ of water and the rack with tubes was covered with a sheet of plastic until the shoots emerged. The tubes were watered with $12 \mathrm{ml}$ of water twice a week and with dilute (1/3 strength) Hoagland's nutrient solution (macro-element only) once a week. The racks were placed in a growth room at $16^{\circ} \mathrm{C}$, with a $16 \mathrm{~h}$ photoperiod. If $G$. graminis var. tritici is present in the ball of roots, the fungus will infect the wheat seedlings. After 3 to 8 weeks, plants were removed from the plastic tubes, and roots were washed to remove the vermiculite and inspected for take-all lesions. Roots with lesions were washed for $2 \mathrm{~h}$ in running tap water and then surface-disinfested by immersion in a solution of $0.5 \%$ silver nitrate for $1 \mathrm{~min}$, followed by rinsing twice in distilled water. Roots were placed on autoclaved Whatman no. 1 filter paper (Whatman Inc., Florham

TABLE 1. Cropping history of fields and geographic origin of Gaeumannomyces graminis var. tritici

\begin{tabular}{|c|c|c|c|c|c|c|c|}
\hline $\begin{array}{l}\text { Location } \\
\text { and isolate }\end{array}$ & Isolate & $\begin{array}{l}\text { Number } \\
\text { of isolates }\end{array}$ & Cropping history & Host & $\begin{array}{l}\text { Presence } \\
\text { of } p h l D^{+} \\
\text {isolates }^{\mathrm{b}}\end{array}$ & $\begin{array}{l}\text { Soil sup- } \\
\text { pressive to } \\
\text { take-all }\end{array}$ & Source \\
\hline Pullman conducive & PC1-13 & 13 & Crop rotation & Wheat & - & - & This study \\
\hline Ritzville TAD & RD $1-6 ; 8-47$ & 46 & 3 years continuous wheat & Wheat & + & + & This study \\
\hline Lind virgin & LV $1-30$ & 30 & Non-cropped & Native grasses & - & - & This study \\
\hline Lind $\mathrm{TAD}^{\mathrm{c}}$ & LD $1-40$ & 40 & 39 years continuous wheat & Wheat & + & + & This study \\
\hline MV116, MV119 & & 2 & & Wheat & & & (24) \\
\hline L109, L116 & & 2 & & Wheat & & & (24) \\
\hline R3-111a-1 & & 1 & & Wheat & & & (24) \\
\hline ARS-A1 & & 1 & & Wheat & & & D. M. Weller, unpublished \\
\hline
\end{tabular}

a The Pullman conducive field was direct-seeded and rain-fed and located on the USDA-ARS Palouse Conservation Field Station. The Almota TAD field was direct-seeded and rain-fed and located on a commercial farm. The Ritzville TAD field was conventionally tilled and irrigated and is located on a commercial farm. The Lind virgin and TAD fields are located on the WSU Lind Dryland Research Station. The Lind TAD field is irrigated and has been both conventionally cultivated and direct-seeded during its history.

${ }^{\mathrm{b}}+$ indicates wheat grown in the soil supports threshold population densities of 2,4-DAPG-producing Pseudomonas fluorescens $\left(>10^{5}\right.$ colony forming units [CFU] root $\left.^{-1}\right)$ (phlD $D^{+}$isolates). - indicates wheat grown in the soil supports population densities of $p_{l} l D^{+}$isolates below the detection limit of $5 \times 10^{3} \mathrm{CFU}$ root ${ }^{-1}$.

${ }^{c}$ LD isolates were from "healthy-looking" wheat plants randomly selected throughout the field. LDP isolates were from wheat plants within distinct patches. 
Park, NJ) to remove excess water. Roots with lesions were cut into pieces $(<1 \mathrm{~cm})$ and placed on R-PDA. Plates were incubated at room temperature in the dark for up to 2 weeks until hyphae grew out from the pieces of root. Hyphae of putative G. graminis var. tritici isolates were selected based on morphological characteristics of hyphae and color change in the medium around the hyphae, and then hyphal tips were transferred to R-PDA. $G$. graminis var. tritici alters the color of rifampicin in R-PDA from orange to purple, and this reaction occurs in as little as $24 \mathrm{~h}$ (14). Over 250 putative isolates of G. graminis var. tritici were isolated and stored as described above.

DNA extraction and Gaeumannomyces graminis varietyspecific polymerase chain reaction. G. graminis var. tritici mycelium was incubated at room temperature for 1 week in $1 / 5 \times$ PDB (BD, Sparks, MD) either in 1.5-ml tubes on a shaker at $150 \mathrm{rpm}$ or in slowly shaken petri dishes. The mycelium was harvested by centrifugation at 5,000 rpm for 5 min and then washed twice in sterile distilled water. DNA was extracted by using a FastDNA Kit (Bio101, Carlsbad, CA) (28) and eluted in $100 \mu \mathrm{l}$ of DNA elution solution. DNA was amplified with $G$. graminis variety-specific primers, NS5 and GGT-RP (16). The polymerase chain reaction (PCR) volume was $50 \mu \mathrm{l}$ : it contained $200 \mu \mathrm{M}$ dNTP mixture, $2 \mathrm{mM} \mathrm{MgCl} 2,10 \mu \mathrm{l}$ of $5 \times$ Green GoTaq Flexi buffer (Promega Corporation, Madison, WI), $0.4 \mu \mathrm{M}$ of each primer; $50 \mathrm{ng}$ of DNA, and 1 unit of Taq polymerase (Promega Corporation, Madison, WI). The PCR was performed in an MJ Research PTC-200 thermal cycler (Bio-Rad, Hercules, CA). An initial denaturation step at $95^{\circ} \mathrm{C}$ for $5 \mathrm{~min}$ was followed by 35 cycles consisting of denaturation at $95^{\circ} \mathrm{C}$ for $45 \mathrm{~s}$, annealing at $52^{\circ} \mathrm{C}$ for $1 \mathrm{~min}$, and extension at $72^{\circ} \mathrm{C}$ for $1 \mathrm{~min}$. After 35 cycles, samples were incubated at $72^{\circ} \mathrm{C}$ for $10 \mathrm{~min}$ for compete extension of PCR products. PCR amplicons were visualized by gel electrophoresis in $1.5 \%$ agarose. A single 410 -bp PCR fragment indicated G. graminis var. tritici, a single 310-bp fragment indicated G. graminis var. avenae, and no PCR product indicated G. graminis var. graminis or a genus other than Gaeumannomyces (16).

To amplify avenacinase-like genes with variety-specific avenacinase-like gene primers (34), the PCR volume was $50 \mu \mathrm{l}$ and contained 25 pmol of each of the three variety-specific 5' primers and 75 pmol of $3^{\prime} \mathrm{AV}-3$ primer. An initial denaturation step at $95^{\circ} \mathrm{C}$ for 5 min was followed by 35 cycles consisting of denaturation at $95^{\circ} \mathrm{C}$ for $45 \mathrm{~s}$, annealing at $64^{\circ} \mathrm{C}$ for $45 \mathrm{~s}$, and extension at $72^{\circ} \mathrm{C}$ for $2 \mathrm{~min}$. To obtain the sequence of partial avenacinase-like genes, PCR products were cleaned using DTR gel filtration cartridges (EdgeBio, Gaithersburg, MD). Sequencing reactions were performed by using a dye-terminator cycle sequencing reaction (ABI-Prism, Foster City, CA) according to the manufacturer's protocol. Reactions were cleaned again using DTR gel filtration cartridges and then completely dried. The reactions were run on an ABI 377 sequencer at the Center for Integrated Biotechnology Bioinformatics Core Facility, Washington State University, Pullman.

In vitro 2,4-DAPG sensitivity assay. G. graminis var. tritici isolates were tested for sensitivity to 2,4-DAPG (Toronto Research Chemicals Inc., Toronto, Canada) in agar plate bioassays. A 4-mm-diameter agar plug was cut with a cork borer from the margin of a 1-week-old culture of an isolate of $G$. graminis var. tritici grown on $1 / 5 \times$ PDA. The agar plug was then transferred with the mycelium facing downward to the center of a 36-mmdiameter well containing $5 \mathrm{ml}$ of $1 / 5 \times$ PDA in a 6-well cell culture cluster (Corning Inc., Corning, NY). Each well contained medium amended with either $0,1,2,3,4$, or $5 \mu \mathrm{g} \mathrm{ml}^{-1}$ of 2,4 DAPG dissolved in methanol. This range of concentrations was used because of the previous findings of Mazzola et al. (24), who demonstrated that $G$. graminis var. tritici isolates that were not inhibited by $3 \mu \mathrm{g} \mathrm{ml}^{-1}$ of 2,4-DAPG were not suppressed on wheat by a phlD ${ }^{+}$strain of $P$. fluorescens. Hyphal growth was measured daily from 3 to 7 days after inoculation. Radial growth was measured from the center of the plug to the edge of the mycelium. Each measurement was performed in four different directions per well. Radial growth measurements were converted to percent growth inhibition by comparing growth on medium amended with the antibiotic to that on medium with no 2,4-DAPG (control, methanol only). Hyphal growth measurement data at day 6 provided the greatest difference between the control and 2,4DAPG treatments, and thus was used for the final analysis of growth inhibition. Growth inhibition data were transformed to probits and linear regression was performed on transformed inhibition data versus 2,4-DAPG concentration using SigmaPlot 8.0 (SPSS Inc., Chicago, IL). Points from all four replicates were pooled together (24 points: four replicates $\times$ six dosages) to run the regression. The $90 \%$ effective dose $\left(\mathrm{ED}_{90}\right)$ values were calculated from the predicted regression equation. Three 2,4-DAPG sensitivity categories (sensitive, moderately sensitive, and less sensitive) were generated based on quartiles from the frequency distribution of $\mathrm{ED}_{90}$ values (1st, 2nd and 3rd combined, and 4th).

Virulence assay. G. graminis var. tritici isolates were tested for virulence on wheat by the tube assay described above except that instead of adding a ball of roots to the assay, two 9-mm agar plugs, cut with a cork borer, from a 1-week-old culture of an isolate grown on 1/5x PDA were added to the column of vermiculite. Virulence tests of each isolate were replicated five times with a replicate consisting of five individual tubes sown with three seeds each. The experiment was arranged in a randomized complete block design. Tubes were incubated and watered as described above. After 4 weeks, plants were removed, washed, and rated for disease on a 0 to 8 scale: $0=$ healthy, $8=$ dead or nearly so (27).

Production of perithecia and measurements of ascospores. To stimulate perithecia formation, plants infected with G. graminis var. tritici were wrapped in wet paper towels, placed in a plastic bag, and incubated in a plant growth room $\left(16^{\circ} \mathrm{C}, 16 \mathrm{~h}\right.$ photoperiod) for 8 to 12 weeks. Perithecia were observed under a dissecting microscope and plants with mature perithecia were washed briefly in sterile water before selecting perithecia. Three perithecia of each isolate were transferred to a microscope glass slide, crushed, and then examined at $\times 40$ and $\times 100$ magnifications with an Olympus BX41TF microscope (Olympus, Center Valley, PA). For each isolate of $G$. graminis var. tritici, pictures of at least 30 ascospores were taken and images were saved as JPEG files. The length of each ascospore was measured using the ImageJ program (available online) (8).

Statistical analysis. The $\mathrm{ED}_{90}$ values for isolates from different locations were analyzed by analysis of variance (ANOVA) and means were separated by Tukey's honestly significant difference test (Statistix 9.0, Analytical Software, St. Paul, MN) to determine if there was any difference in sensitivity to 2,4-DAPG among isolates from different locations. Disease severity of isolates from the various locations was compared by ANOVA, and means were separated by a protected least significant difference test $(P=0.05)$ by SAS 9.1.3 (SAS Institute, Inc., Cary, NC). Linear regression analyses (SigmaPlot 8.0) were performed to determine the relationship between the $\mathrm{ED}_{90}$ of 2,4-DAPG and the virulence of G. graminis var. tritici isolates.

\section{RESULTS}

Isolation of G. graminis var. tritici from fields with different cropping histories. G. graminis var. tritici was isolated from roots of grasses from noncropped virgin sites and from wheat grown in fields that had undergone crop rotation or three or more years of continuous wheat. Roots of wheat grown in soil from the Pullman conducive and Lind virgin fields do not contain threshold population densities of 2,4-DAPG-producing $P$. fluorescens ( $>10^{5} \mathrm{CFU} \mathrm{\textrm {g } ^ { - 1 }}$ of root) (density needed to suppress take-all; 32), whereas soils from the other fields contained threshold densities (Table 1). Over 250 putative G. graminis var. tritici isolates were 
isolated from roots on R-PDA and tentatively identified as $G$. graminis var. tritici on the basis of a color change on R-PDA and morphological characteristics such as colony color and curling back of the hyphae on the edge of the colony (14). On R-PDA, the color of the medium changes from orange to purple around the $G$. graminis var. tritici mycelium. We focused on 177 new $G$. graminis var. tritici isolates: 134 isolates from fields with three or more years of continuous wheat or barley, 13 isolates from the Pullman take-all conducive field, and 30 isolates from the Lind noncropped (virgin) soil (Table 1).

Identification of $G$. graminis var. tritici by morphological characteristics and PCR. Of the 183 isolates used in this study (177 new and 6 control isolates), 135 were tested for ability to produce perithecia. Perithecia characteristic of $G$. graminis var. tritici, were produced by 119 isolates on infected wheat roots (cv. Penawawa), but were not formed by the other isolates after 12 weeks of incubation. Ascospore length ranged from 61 to $90 \mu \mathrm{m}$ (average $78.3 \mu \mathrm{m}$ ) (data not shown), which is typical of $G$. graminis var. tritici. Among the isolates, the color of the hyphae and the growth habit on 1/5× PDA (data not shown) varied, which is also typical of $G$. graminis var. tritici (22). We also observed simple hyphopodia on the outer layers of wheat seedling coleoptiles (data not shown) produced by control (ARS-A1, R3-111a-1) and new (i.e., ADB-15) isolates of $G$. graminis var. tritici.

We then performed $G$. graminis variety-specific PCR with NS5 and GGT-RP primers, which amplify $18 \mathrm{~S}$ ribosomal DNA of $G$. graminis. All 183 G. graminis var. tritici isolates used in this study generated a single 410-bp PCR product indicative of var. tritici (Fig. 1).

It was previously reported (34) that nucleotide differences in avenacinase-like genes can distinguish among G. graminis vars. avenae, graminis, and tritici based upon the size of PCR amplicons. All of the $183 \mathrm{G}$. graminis var. tritici isolates from Washington State responded positively with G. graminis varietyspecific mixed primers (each of the three variety-specific primers and the $3^{\prime}$ common primer). The size of the PCR amplicons was approximately 1,100-bp (data not shown). Because Rachawong et al. (34) reported that amplification of the var. tritici and var. graminis avenacinase-like genes generated 870- and 1,086-bp PCR products, respectively, we sequenced the primer's binding site of the avenacinase-like genes from six of our G. graminis var. tritici isolates (Fig. 2A and B) in order to determine the basis of the discrepancy. The var. tritici-specific primer sequence is 5TCCTCGGCCCCGTAATTGGC-3. Compared with the isolates of Rachdawong et al. (34), Washington State isolates had two different nucleotides in the primer binding site ( $\mathrm{C}$ to $\mathrm{T}$ ). However, the primer binding site sequences were identical in the Washington isolates and the American Type Culture Collection (ATCC, Manassas, VA) type strain ATCC28230 (Fig. 2B). Unexpectedly, the sequence of the var. graminis-specific primer (5-ACCCCCGGTCCCTGCGTA-3) was complementary to the binding site of the avenacinase-like gene in $G$. graminis var. tritici isolates from Washington and strain ATCC28230 (Fig. 2A). As a result, PCR with the $G$. graminis variety-specific primers generated amplicons of approximately $1,100 \mathrm{bp}$ with Washington isolates.

Sensitivity of G. graminis var. tritici to 2,4-DAPG. Of the 183 G. graminis var. tritici isolates used in this study, 155 were tested for sensitivity to 2,4-DAPG (Fig. 3). Regardless of source, isolates varied in sensitivity to 2,4-DAPG. In all soils, sensitivity of isolates to 2,4-DAPG was normally distributed. For isolates from all soils, the range of $\mathrm{ED}_{90}$ of 2,4-DAPG was 3.1 to $11.1 \mu \mathrm{g}$ $\mathrm{ml}^{-1}$. Based on $\mathrm{ED}_{90}$ frequency distributions, G. graminis var. tritici isolates were divided into three sensitivity categories: sensitive $\left(\mathrm{ED}_{90} ; 3.1\right.$ to $\left.4.4 \mu \mathrm{g} \mathrm{ml}^{-1}\right)$ (37 isolates), moderately sensitive $\left(\mathrm{ED}_{90} ; 4.5\right.$ to $\left.6.1 \mu \mathrm{g} \mathrm{ml}^{-1}\right)$ ( 75 isolates), and less sensitive ( $\mathrm{ED}_{90}$; 6.2 to $11.1 \mu \mathrm{g} \mathrm{ml}^{-1}$ ) (37 isolates). The ratio of sensitive, moderately sensitive, and less sensitive isolates was approximately 1:2:1. The average $\mathrm{ED}_{90}$ was $4.6 \mu \mathrm{g} \mathrm{ml}^{-1}$ for ADB isolates; $5.8 \mu \mathrm{g}$ $\mathrm{ml}^{-1}$ for LD isolates; $5.8 \mu \mathrm{g} \mathrm{ml}^{-1}$ for LDP isolates; $5.1 \mu \mathrm{g} \mathrm{ml}^{-1}$ for $\mathrm{LV}$ isolates; $5.5 \mu \mathrm{g} \mathrm{m}^{-1}$ for PC isolates; and $5.3 \mu \mathrm{g} \mathrm{ml}^{-1}$ for RD isolates. Sensitivity of isolates to 2,4-DAPG was not correlated with cropping history (Fig. 4). Six isolates were exceptions to the above pattern: ADB-11 was insensitive to 2,4-DAPG $\left(\mathrm{ED}_{90}>11.1\right.$ $\mu \mathrm{g} \mathrm{ml}^{-1}$ ) and isolates ADB-14, LD-5, LDP-14, LDP-21, and LV-2 were extremely sensitive to 2,4-DAPG $\left(\mathrm{ED}_{90}<1 \mu \mathrm{g} \mathrm{ml} \mathrm{m}^{-1}\right)$. Exact $\mathrm{ED}_{90}$ values for these isolates were not calculated because they were outside of the range of linear regression of growth inhibition. These isolates are the focus of another ongoing study.

Virulence of G. graminis var. tritici isolates. Of the $183 G$. graminis var. tritici isolates used in this study, 135 were tested for virulence on wheat (cv. Penawawa). Most of these isolates caused significant disease. Exceptions included the eight isolates LD-27, LDP-14, LD-28, ADB-14, ADB-11, LD-24, LD-33, and PC-2, which had disease ratings of less than 1 . Disease ratings ranged from 0 to 5.78. Virulence tests divided G. graminis var. tritici isolates into three groups based on the frequency distribution, weakly virulent (rating 0 to 2.83 ) (34 isolates), moderately virulent (rating 2.84 to 4.19 ) (67 isolates), and strongly virulent (rating 4.2 to 5.78 ) (34 isolates); the ratio was approximately $1: 2: 1$, respectively. Populations of isolates from different locations did not differ significantly in virulence (Fig. 5). In addition, linear regression analyses showed that among G. graminis var. tritici isolates from Washington State fields there was no correlation between virulence (as determined in the tube assay) and sensitivity to 2,4-DAPG in vitro (Fig. 6).

\section{DISCUSSION}

In the Pacific Northwest and The Netherlands, TAD develops because of the buildup of populations of 2,4-DAPG-producing $P$. fluorescens and production of the antibiotic in the wheat or barley rhizosphere (38). We frequently have been asked whether isolates of $G$. graminis var. tritici with reduced sensitivity to 2,4-DAPG become enriched in monoculture wheat and barley fields as a

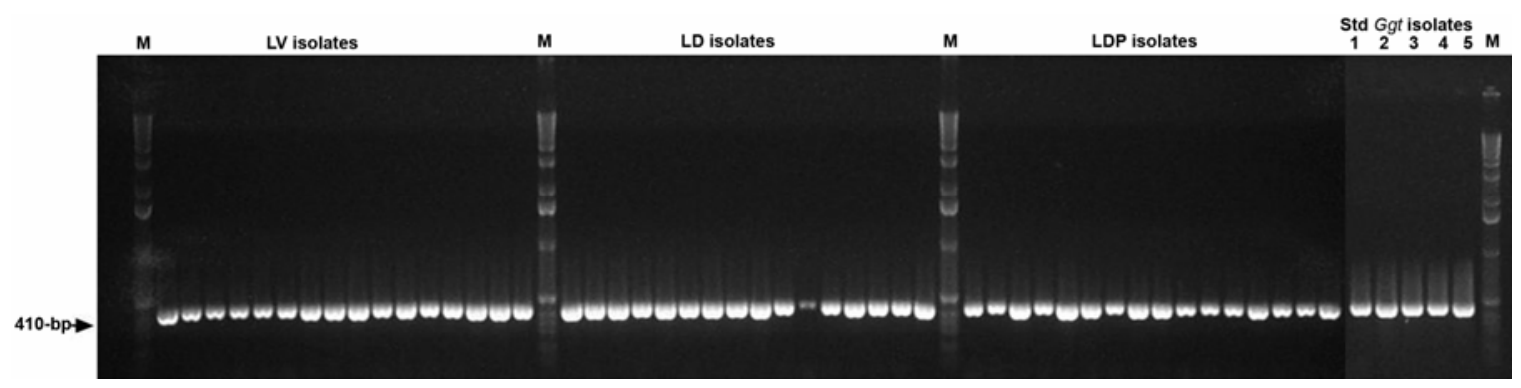

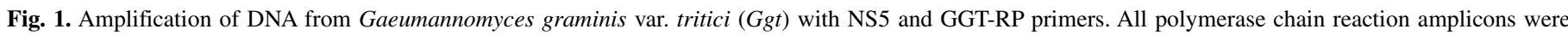

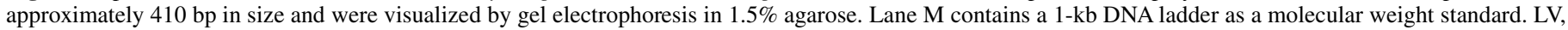

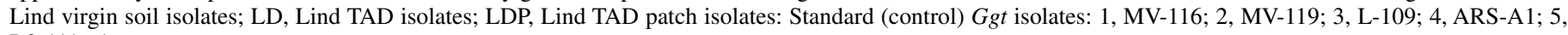
R3-111a-1. 
result of exposure to the antibiotic. To address this question, we compared the $\mathrm{ED}_{90}$ for 2,4-DAPG against isolates of G. graminis var. tritici from well-studied TAD and non-TAD fields. $\mathrm{ED}_{90}$ values for all isolates tested ranged from 3.1 to $11.1 \mu \mathrm{g} \mathrm{ml}^{-1}$, and like Mazzola et al. (24), we found that isolates showed differential sensitivity to 2,4-DAPG. However, sensitivity to the antibiotic was normally distributed among isolates regardless of the source (monoculture versus nonmonoculture fields), and the average $\mathrm{ED}_{90}$ values for isolates from the fields tested did not differ statistically with the exception of ADB isolates, which had a lower average $\mathrm{ED}_{90}$ value than those of the LD and LDP isolates (Fig. 4).

Of particular interest were the results from Lind because the Lind TAD and virgin fields are located only about $100 \mathrm{~m}$ apart. The average $\mathrm{ED}_{90}$ for isolates from the non-cropped Lind virgin site (no detectable 2,4-DAPG producers) was $5.1 \mu \mathrm{g} \mathrm{ml} \mathrm{ml}^{-1}$, whereas the average $\mathrm{ED}_{90}$ for isolates from the Lind TAD field (threshold $p h l D^{+}$population) was $5.8 \mu \mathrm{g} \mathrm{ml}^{-1}$. Thus, after 39 years of continuous wheat monoculture, significant changes in sensitivity to 2,4-DAPG of isolates in the Lind TAD field had not occurred. In addition, within the Lind TAD field, the average $\mathrm{ED}_{90}$ for isolates inside and outside of take-all patches was identical, suggesting that the patches do not result from pathogen isolates that are less sensitive to 2,4-DAPG. TAD effectively suppresses the development of severe take-all in the field, but the robustness of its expression varies among fields and years. Fluctuations in take-all incidence and severity are a normal but poorly understood part of this dynamic process (22). In Washington TAD fields, small take-all patches typically occur even in strongly suppressive fields. In years when TAD is weakly expressed, patches are much larger and more numerous.

Collectively, our results indicate that (i) differential sensitivity to 2,4-DAPG is a natural characteristic of G. graminis var. tritici populations, (ii) production of 2,4-DAPG in the rhizosphere does not exert a selection pressure on G. graminis var. tritici in Washington State TAD fields, and (iii) isolates with reduced sensitivity to the antibiotic do not appear to emerge in a field even after decades of wheat monoculture and exposure to the antibiotic. To our knowledge, this is the first study addressing the question of emergence of resistance in a target pathogen to an introduced or indigenous biocontrol agent.

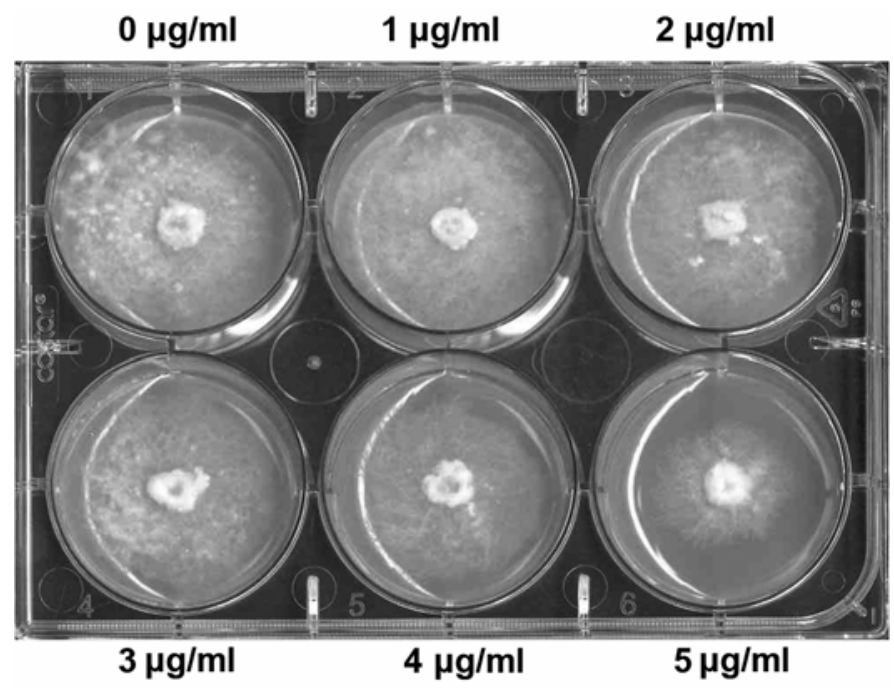

Fig. 3. Six-well plate assay used to test the sensitivity of isolates of Gaeumannomyces graminis var. tritici to 2,4-diacetylphloroglucinol (2,4-DAPG). 1/5× potato dextrose agar was amended with either $0,1,2,3,4$, or $5 \mu \mathrm{g}$ of $2,4-$ DAPG $\mathrm{ml}^{-1}$. A plug of the fungus was placed in the center of the well. Radial growth of each isolate well was measured over a period of 3 to 7 days after inoculation. The picture was taken at 6 days after inoculation.
A

Ggg Primer sequence
Ggt_CB1
Ggt_M1
Ggt_CH1
Ggg_ATCC12761
Ggt_ATCC28230
Gga_ATCC15419
ADB11
ARS-A1
R3-111a-1
ADB3
PC1
LDP21

B

Ggt Primer sequence
Ggt_CB1
Ggt_CH1
Ggg_ATCC12761
Ggt_ATCC28230
Gga_ATCC15419
ADB11
ARS-A1
R3-111a-1
ADB3
PC1
LDP21

5-ACCCCCGGTCCCTGCGTA-3 CGGGCACCCCCGGTCCCTGCGTGGGCAACATCGCCCCCGTGCCGCGCCTC CGGGCACCCCCGGTCCCTGCGTGGGCAACATCGCCCCCGTGCCGCGCCTC CGGGCACCCCCGGTCCCTGCGTGGGCAACATCGCCCCCGTGCCGCGCCTC CGGGCACCCCCGGTCCCTGCGTAAGCAACATCGCCCCCGTGCCGCGCCTC CGGGCACCCCCGGTCCCTGCGTAGGCAACATCGCCCCCGTGCCGCGCCTC CAGGCACCCCCGGTCCCTGCGTGGGCAACATCGCCCCCGTGCCGCGCCTC CGGGCACCCCCGGTCCCTGCGTAGGCAACATCGCCCCCGTGCCGCGCCTC CGGGCACCCCCGGTCCCTGCGTAGGCAACATCGCCCCCGTGCCGCGCCTC CGGGCACCCCCGGTCCCTGCGTAGGCAACATCGCCCCCGTGCCGCGCCTC CGGGCACCCCCGGTCCCTGCGTAGGCAACATCGCCCCCGTGCCGCGCCTC CGGGCACCCCCGGTCCCTGCGTAGGCAACATCGCCCCCGTGCCGCGCCTC CGGGCACCCCCGGTCCCTGCGTAGGCAACATCGCCCCCGTGCCGCGCCTC

\section{5-TCCTCGGCCCCGTAATTGGC-3}

GACAAGGGCTCTCACATCATCCTCGGCCCCGTAATTGGCCCCCTTGGAAG GACAAGGGCTCTCACATCATCCTCGGCCCCGTAATTGGCCCCCTTGGAAG GACAAGGGCTCTCACATCATCCTCGGCCCTGTAATTGGTCCCCTTGGAAG GACAAGGGCTCTCACATCATCCTCGGCCCTGTAATTGGTCCCCTTGGAAG GACAAGGGGTCCCACGTCATCCTCGGCCCTGTAATTGGTCCCCGTGGAAG GACAAGGGCTCTCACATCATCCTCGGCCCTGTAATTGGTCCCCTTGGAAG GACAAGGGCTCTCACATCATCCTCGGCCCTGTAATTGGTCCCCTTGGAAG GACAAGGGCTCTCACATCATCCTCGGCCCTGTAATTGGTCCCCTTGGAAG GACAAGGGCTCTCACATCATCCTCGGCCCTGTAATTGGTCCCCTTGGAAG GACAAGGGCTCTCACATCATCCTCGGCCCTGTAATTGGTCCCCTTGGAAG GACAAGGGCTCTCACATCATCCTCGGCCCTGTAATTGGTCCCCTTGGAAG

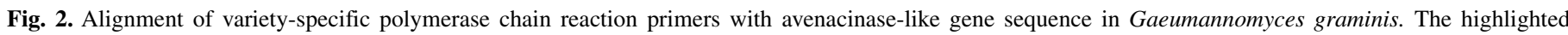

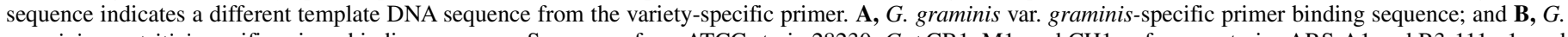

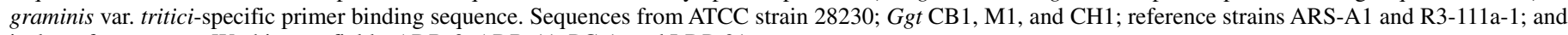
isolates from eastern Washington fields, ADB-3, ADB-11, PC-1, and LDP-21. 
Several factors may explain why isolates of G. graminis var. tritici with reduced sensitivity to 2,4-DAPG have not emerged or become enriched in Washington State TAD soils. For example, the pathogen may be exposed to inhibitory concentrations of the antibiotic only during its parasitic phase in sites, such as infection courts and lesions, where $p h l D^{+}$bacteria proliferate. During the rest of the pathogen's life cycle, while growing saprophytically or surviving in crown and root tissues, 2,4-DAPG may not be present or is present at extremely low levels. This scenario would reduce the selection pressure on $G$. graminis var. tritici for the emergence of isolates with less sensitivity to the antibiotic, as compared to if the pathogen was exposed to the antibiotic throughout its life cycle.

Regression analysis revealed no correlation between antibiotic sensitivity and virulence (Fig. 6), suggesting that reduced sensitivity to 2,4-DAPG does not affect the pathogen's virulence. However, the impact of reduced antibiotic sensitivity on the saprophytic growth and survival of $G$. graminis var. tritici has not been assessed. Elucidating the fine details about the spatial and temporal accumulation and subsequent degradation of 2,4-DAPG in the rhizosphere environment and in plant debris will help to clarify the extent to which the pathogen is exposed to the antibiotic. However, quantifying antibiotics in the rhizosphere of field-grown plants currently requires extractions from multiple pooled root systems, and detecting 2,4-DAPG in individual microsites is currently beyond the capacity of commonly used bioanalytical techniques.

A second factor may relate to the wheat or barley varieties grown a TAD field. Washington State growers often change wheat varieties for economic reasons or pest control. It is well documented that both the crop species and varieties (including of wheat) differentially enrich and support $p h l D^{+}$populations $(4,13$, 25 ) and genotypes $(23,25,30)$, and modulate $2,4-\mathrm{DAPG}$ accumulation in the rhizosphere $(4,26)$. For example, Okubara and Bonsall (26) reported significant differences in the accumulation of 2,4-DAPG on the roots of wheat cultivars Tara, Buchanan, and Finley by $P$. fluorescens Q8r1-96, a strain typical of those primarily responsible for TAD in Washington State (38). It is

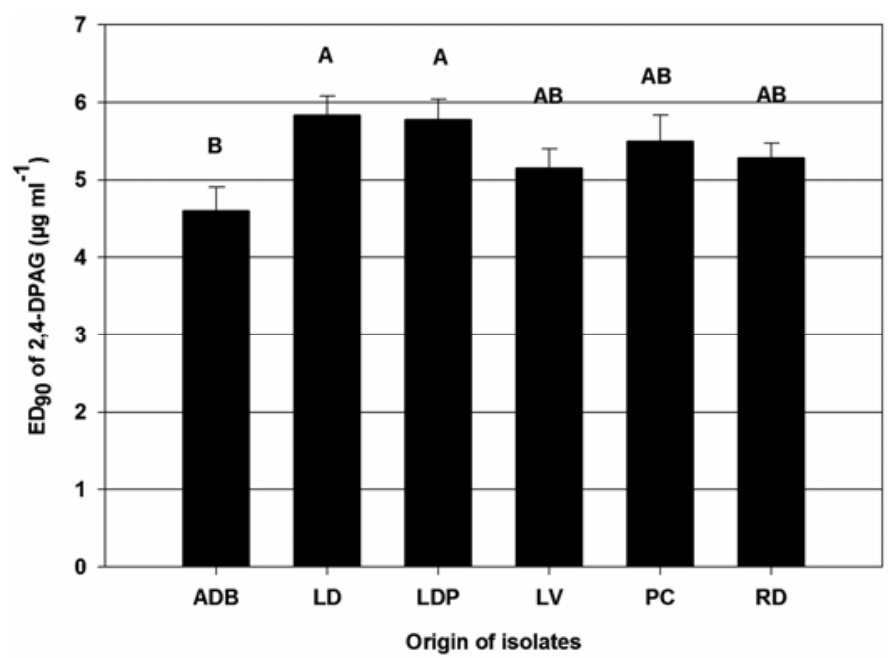

Fig. 4. The $90 \%$ effective dose $\left(\mathrm{ED}_{90}\right)$ value of 2,4-diacetylphloroglucinol (2,4-DAPG) against isolates of Gaeumannomyces graminis var. tritici from fields in Washington State with different cropping histories. The $\mathrm{ED}_{90}$ of 2,4DAPG was calculated on the basis of the inhibition of radial growth of $G$. graminis var. tritici on $1 / 5 \times$ potato dextrose agar amended with a range of concentrations of 2,4-DAPG. Source of isolates and number tested: ADB, Almota TAD (18 isolates); LD, Lind TAD (25 isolates); LDP, Lind TAD patch (24 isolates); LV, Lind virgin (25 isolates); PC, Pullman conducive (13 isolates); and RD, Ritzville TAD (44 isolates). Bars with the same letters are not significantly different according to Tukey's honestly significant difference test $(P=0.05)$. likely, therefore, that the concentration of the antibiotic to which isolates of G. graminis var. tritici are exposed in the field can vary significantly from year to year due to changes in varieties. In addition, we think that the supportiveness of a variety to 2,4DAPG producers and antibiotic accumulation is an important contributor to the robustness of the suppressiveness observed in a TAD field.

Finally, the mechanism of action of 2,4-DAPG may hamper emergence of less sensitive isolates in the field. Although the exact mechanism of action of 2,4-DAPG is not known, the antibiotic has antiviral, antibacterial, antifungal, antihelminthic and phytotoxic properties (38). Thus, given the breadth of its activity

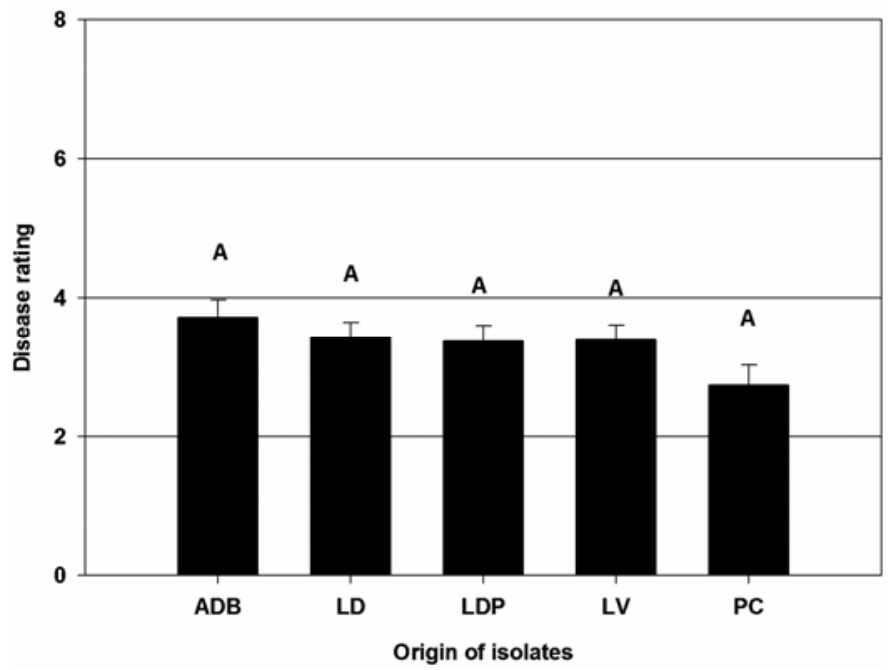

Fig. 5. Severity of take-all on wheat caused by isolates of Gaeumannomyces graminis var. tritici from fields in central and eastern Washington State. Disease was measured by the tube assay and plants were incubated in a growth room $\left(16^{\circ} \mathrm{C}, 16 \mathrm{~h}\right.$ photoperiod). Take-all was rated at 4 weeks on a 0 to 8 scale. Source of isolates and number tested: ADB, Almota TAD (18 isolates); LD, Lind TAD (39 isolates); LDP, Lind TAD patch (29 isolates); LV, Lind virgin (30 isolates); and PC, Pullman conducive (13 isolates). Bars with the same letters are not significantly different according to the least significant difference test $(P=0.05)$.

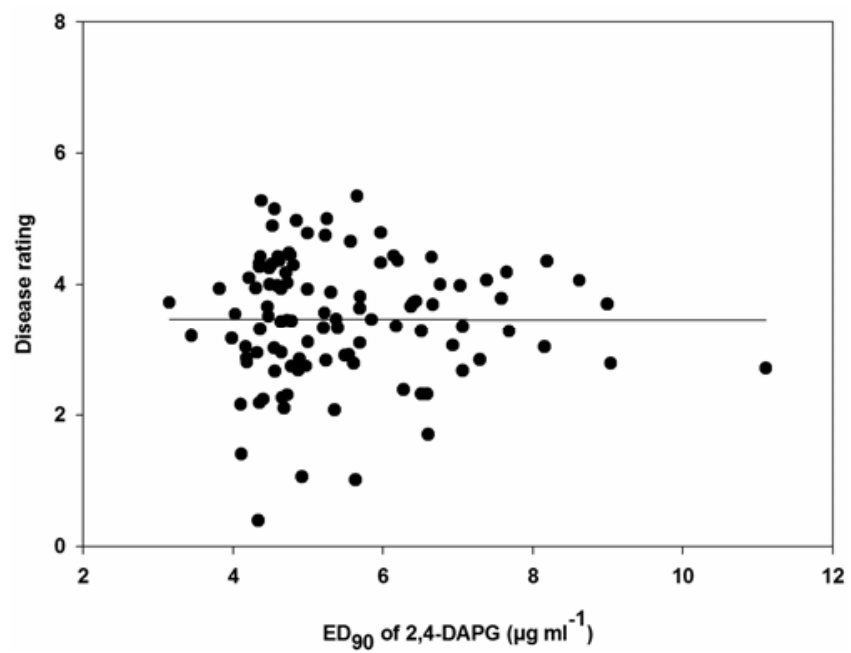

Fig. 6. Relationship between disease ratings on wheat (cv. Penawawa) and sensitivity to 2,4-diacetylphloroglucinol (2,4-DAPG) of isolates of Gaeumannomyces graminis var. tritici from fields in central and eastern Washington State. Isolates were tested in the tube assay and take-all severity was rated on a 0 to 8 scale. G. graminis var. tritici isolates were grown on $1 / 5 \times$ potato dextrose agar amended with a range of concentrations of 2,4-DAPG (0 to $5 \mu \mathrm{g}$ $\mathrm{ml}^{-1}$ ) and radial growth was measured daily. The $90 \%$ effective dose was calculated by the Probit analysis function in SigmaPlot 8.0. Correlation coefficient: $r^{2}=0.00498 ; P=0.9573$. 
against such a wide range of organisms, it is not likely that 2,4DAPG has a single site of action. This would reduce the probability of mutants with less sensitivity emerging in the $G$. graminis var. tritici population.

Identification of $G$. graminis varieties on the basis of pathogenicity tests and morphological characteristics can be time consuming and sometimes inconclusive because of overlap in characteristics with G. graminis var. avenae (attacks oat and turf grasses), var. graminis (attacks Bermuda grass, rice and other grasses) and Phialophora $(19,22)$. G. graminis var. graminis and var. tritici have similar ascospore length, ranging from 70 to $105 \mu \mathrm{m}$, whereas G. graminis var. avenae has slightly longer ascospores $(100$ to $130 \mu \mathrm{m})$. G. graminis var. avenae and tritici produce simple hyphopodia on infected plant tissues, whereas var. graminis produces lobed hyphopodia $(19,22)$. For example, Yeates et al. (40) identified G. graminis var. tritici isolates based on ascospore length, yet they attacked oat.

Several molecular approaches have been developed to facilitate identification of varieties of $G$. graminis: i.e., restriction fragment length polymorphism using a mitochondrial DNA probe $(3,20$, $21)$; restriction patterns of PCR products using internal transcribed spacer (ITS) region and 18S rDNA (17,37); random amplified polymorphic DNA $(6,18)$; and specific PCR assays based on nuclear rDNA $(5,16)$ or avenacinase and avenacinaselike gene sequences (34). However, some of these molecular methods may not be reliable across a broad collection of isolates (19). We conducted PCR on DNA of our isolates with NS5 and GGT-RP primers, which are reported to amplify $18 \mathrm{~S}$ ribosomal DNA of G. graminis var. tritici as a single 410-bp fragment (16). Our isolates, which caused typical take-all symptoms and had hyphal characteristics, growth on R-PDA, and ascospores typical of G. graminis var. tritici, generated a single 410-bp PCR product. Thus, we are confident that the isolates selected for our studies were typical G. graminis var. tritici.

Rachdawong et al. (34) reported sequence variation in the avenacinase-like gene in $G$. graminis varieties that could distinguish vars. avenae, graminis, and tritici. They developed three variety-specific forward primers and one common reverse primer (AV3). Varieties were identified based on different PCR amplicons sizes: $617 \mathrm{bp}$ for $G$. graminis var. avenae, $870 \mathrm{bp}$ for var. tritici, and 1,086 bp for var. graminis. We tested these primers early in our study but unexpectedly found that DNA from our new G. graminis var. tritici isolates from Washington and six control isolates generated PCR amplicons of approximately 1,100 bp in size with a mix of the three variety-specific primers. Alignment of the sequences of the avenacinase-like genes of different isolates showed variation, especially in the region of the binding site of the G. graminis variety-specific primers (Fig. 2). Washington isolates have nucleotide variation in the avenacinase-like gene as compared to isolates tested by Rachdawong et al. (34). These sequence variations resulted in the 1,100-bp PCR amplicons from the Washington G. graminis var. tritici isolates. However, Washington isolates had the same sequence as G. graminis var. tritici type strain ATCC28230 (Fig. 2). In general, our results highlight the need to test a broad range of $G$. graminis var. tritici isolates from multiple locations when developing specific primers for $G$. graminis. We suggest that at least two different molecular methods be employed to identify a $G$. graminis variety.

\section{ACKNOWLEDGMENTS}

This research was supported by a grant from the Washington State University, O. A. Vogel Wheat Research Grants Program. P. A. H. M. Bakker was a recipient of a fellowship from the Organisation for Economic Co-operation and Development (OECD). We thank K. Weller for his technical support and O. Mavrodi, K. L. Schroeder, and L. Thomashow for discussions, contributions, and comments on the manuscript.

\section{LITERATURE CITED}

1. Allende-Molar, R. 2006. Role of 2,4-diacetylphloroglucinol-producing Pseudomonas fluorescens in the suppression of take-all and Pythium root rot of wheat. Ph.D. thesis. Department of Plant Pathology. Washington State University, Pullman.

2. Bangera, M. G., and Thomashow, L. S. 1999. Identification and characterization of a gene cluster for synthesis of the polyketide antibiotic 2,4-diacetylphloroglucinol from Pseudomonas fluorescens Q2-87. J. Bacteriol. 181:3155-3163.

3. Bateman, G. L., Ward, E., Hornby, D., and Gutteridge, R. J. 1997. Comparisons of isolates of the take-all fungus, Gaeumannomyces graminis var. tritici, from different cereal sequences using DNA probes and non-molecular methods. Soil Biol. Biochem. 29:1225-1232.

4. Bergsma-Vlami, M., Prins, M. E., and Raaijmakers, J. M. 2005. Influence of plant species on population dynamics, genotypic diversity and antibiotic production in the rhizosphere by indigenous Pseudomonas spp. FEMS Microbiol. Ecol. 52:59-69.

5. Bryan, G. T., Daniels, M. J., and Osbourn, A. E. 1995. Comparison of fungi within the Gaeumannomyces-Phialophora complex by analysis of ribosomal DNA sequences. Appl. Environ. Microbiol. 61:681-689.

6. Bryan, G. T., Labourdette, E., Melton, R. E., Nicholson, P., Daniels, M. J., and Osbourn, A. E. 1999. DNA polymorphism and host range in the takeall fungus, Gaeumannomyces graminis. Mycol. Res. 103:319-327.

7. Colbach, N., Lucas, P., and Meynard, J. M. 1997. Influence of crop management on take-all development and disease cycles on winter wheat. Phytopathology 87:26-32.

8. Collins, T. J. 2007. ImageJ for microscopy. Biotechniques 43(1 suppl.): 25-30.

9. Cook, R. J. 2003. Take-all of wheat. Physiol. Mol. Plant Pathol. 62:73-86.

10. Cook, R. J. 2007. Take-all decline: Model system in the science of biological control and clue to the success of intensive cropping. Pages 399-414 in: Biological Control a Global Perspective: Case Studies from Around the World. C. Vincent, M. Goettel, and G. Lazarovits, eds. CAB International, Wallingford, UK.

11. Cook, R. J., and Naiki, T. 1982. Virulence of Gaeumannomyces graminis var. tritici from fields under short-term and long-term wheat cultivation in the Pacific Northwest, USA. Plant Pathol. 31:201-207.

12. Cook, R. J., and Rovira, A. D. 1976. The role of bacteria in the biological control of Gaeumannomyces graminis by suppressive soils. Soil Biol. Biochem. 8:269-273.

13. De La Fuente, L., Landa, B. B., and Weller, D. M. 2006. Host crop affects rhizosphere colonization and competitiveness of 2,4-diacetylphloroglucinol-producing Pseudomonas fluorescens. Phytopathology 96:751762.

14. Duffy, B. K., and Weller, D. M. 1994. A semiselective and diagnostic medium for Gaeumannomyces graminis var. tritici. Phytopathology 84:1407-1415.

15. Elliott, M. L. 2005. Survival, growth and pathogenicity of Gaeumannomyces graminis var. graminis with different methods of long-term storage. Mycologia 97:901-907.

16. Fouly, H. M., and Wilkinson, H. T. 2000. Detection of Gaeumannomyces graminis varieties using polymerase chain reaction with variety-specific primers. Plant Dis. 84:947-951.

17. Fouly, H. M., Wilkinson, H. T., and Chen, W. D. 1997. Restriction analysis of internal transcribed spacers and the small subunit gene of ribosomal DNA among four Gaeumannomyces species. Mycologia 89: 590-597.

18. Fouly, H. M., Wilkinson, H. T., and Domier, L. L. 1996. Use of random amplified polymorphic DNA (RAPD) for identification of Gaeumannomyces species. Soil Biol. Biochem. 28:703-710.

19. Freeman, J., and Ward, E. 2004. Gaeumannomyces graminis, the take-all fungus and its relatives. Mol. Plant Pathol. 5:235-252.

20. Henson, J. M. 1989. DNA probe for identification of the take-all fungus, Gaeumannomyces graminis. Appl. Environ. Microbiol. 55:284-288.

21. Henson, J. M. 1992. DNA hybridization and polymerase chain reaction (PCR) tests for identification of Gaeumannomyces, Phialophora and Magnaporthe isolates. Mycol. Res. 96:629-636.

22. Hornby, D. 1998. Take-All of Cereals. A Regional Perspective. CAB International, Wallingford, UK.

23. Landa, B. B., Mavrodi, O. V., Schroeder, K. L., Allende-Molar, R., and Weller, D. M. 2006. Enrichment and genotypic diversity of phlDcontaining fluorescent Pseudomonas spp. in two soils after a century of wheat and flax monoculture. FEMS Microbiol. Ecol. 55:351-368.

24. Mazzola, M., Fujimoto, D. K., Thomashow, L. S., and Cook, R. J. 1995. Variation in sensitivity of Gaeumannomyces graminis to antibiotics produced by fluorescent Pseudomonas spp. and effect on biological control of take-all of wheat. Appl. Environ. Microbiol. 61:2554-2559.

25. Mazzola, M., Funnell, D. L., and Raaijmakers, J. M. 2004. Wheat cultivar-specific selection of 2,4-diacetylphloroglucinol-producing fluo- 
rescent Pseudomonas species from resident soil populations. Microbiol. Ecol. 48:338-348.

26. Okubara, P. A., and Bonsall, R. F. 2008. Accumulation of Pseudomonasderived 2,4-diacetylphloroglucinol on wheat seedling roots is influenced by host cultivar. Biol. Control. 46:322-331.

27. Ownley, B. H., Weller, D. M., and Thomashow, L. S. 1992. Influence of in situ and in vitro $\mathrm{pH}$ on suppression of Gaeumannomyces graminis var. tritici by Pseudomonas fluorescens 2-79. Phytopathology 82:178-184.

28. Paulitz, T. C., and Adams, K. 2003. Composition and distribution of Pythium communities in wheat fields in eastern Washington State. Phytopathology 93:867-873.

29. Paulitz, T. C., Smiley, R. W., and Cook, R. J. 2002. Insights into the prevalence and management of soil borne cereal pathogens under direct seeding in the Pacific Northwest, U.S.A. Can. J. Plant Pathol. 24:416-428.

30. Picard, C., Frascaroli, E., and Bosco, M. 2004. Frequency and biodiversity of 2,4-diacetylphloroglucinol-producing rhizobacteria are differentially affected by the genotype of two maize inbred lines and their hybrid. FEMS Microbiol. Ecol. 49:207-215.

31. Raaijmakers, J. M., Bonsall, R. E., and Weller, D. M. 1999. Effect of population density of Pseudomonas fluorescens on production of 2,4diacetylphloroglucinol in the rhizosphere of wheat. Phytopathology 89:470-475.

32. Raaijmakers, J. M., and Weller, D. M. 1998. Natural plant protection by 2,4-diacetylphloroglucinol-producing Pseudomonas spp. in take-all decline soils. Mol. Plant-Microbe. Interact. 11:144-152.
33. Raaijmakers, J. M., Weller, D. M., and Thomashow, L. S. 1997. Frequency of antibiotic-producing Pseudomonas spp. in natural environments. Appl. Environ. Microbiol. 63:881-887.

34. Rachdawong, S., Cramer, C. L., Grabau, E. A., Stromberg, V. K., Lacy, G. H., and Stromberg, E. L. 2002. Gaeumannomyces graminis vars. avenae, graminis, and tritici identified using PCR amplification of avenacinaselike genes. Plant Dis. 86:652-660.

35. Shipton, P. J. 1972. Take-all in spring-sown cereals under continuous cultivation: Disease progress and decline in relation to crop succession and nitrogen. Ann. Appl. Biol. 71:33-46.

36. Smiley, R. W. 1979. Wheat-rhizoplane pseudomonads as antagonists of Gaeumannomyces graminis. Soil Biol. Biochem. 11:371-376.

37. Tan, M. K. 1997. Origin and inheritance of group I introns in $26 \mathrm{~S}$ rRNA genes of Gaeumannomyces graminis. J. Mol. Evol. 44:637-645

38. Weller, D. M., Landa, B. B., Mavrodi, O. V., Schroeder, K. L., De La Fuente, L., Bankhead, S. B., Allende-Molar, R., Bonsall, R. F., Mavrodi, D. V., and Thomashow, L. S. 2007. Role of 2,4-diacetylphloroglucinolproducing fluorescent Pseudomonas spp. in the defense of plant roots. Plant Biol. 9:4-20.

39. Weller, D. M., Raaijmakers, J. M., McSpadden Gardener, B. B., and Thomashow, L. S. 2002. Microbial populations responsible for specific soil suppressiveness to plant pathogens. Annu. Rev. Phytopathol. 40:309348.

40. Yeates, J. S., Fang, C. S., and Parker, C. A. 1986. Distribution and importance of oat-attacking isolates of Gaeumannomyces graminis var. tritici in western Australia. Trans. Br. Mycol. Soc. 86:145-152. 